\title{
Uso de uma Ontologia de Fundamentação para Dirimir Ambiguidades na Modelagem de Processos de Negócio
}

\author{
Aline F. Martins, Ricardo A. Falbo, Giancarlo Guizzardi, João Paulo A. Almeida \\ Núcleo de Estudos em Modelagem Conceitual e Ontologias (NEMO) - Departamento \\ de Informática - Universidade Federal do Espírito Santo (UFES) \\ Av. Fernando Ferrari s/n, Campus de Goiabeiras - 29.060-900 - Vitória - ES - Brasil \\ \{falbo, gguizzardi, jpalmeida\}@inf.ufes.br
}

\begin{abstract}
Resumo. Modelos de processos de negócio são usados, dentre outros, para aperfeiçoar a operação das empresas e para apoiar o desenvolvimento de sistemas de informação. Neste contexto a qualidade dos modelos é essencial. Modelos de processo ambíguos e mal formados podem levar a problemas de entendimento, comprometendo seu uso. Um aspecto importante para a qualidade de modelos é a clareza e expressividade da linguagem de modelagem utilizada. Este artigo explora o uso da Ontologia de Fundamentação Unificada (Unified Foundational Ontology - UFO) para adicionar semântica a alguns elementos de modelo de diagramas de atividade da UML, de modo capturar distinções importantes e tornar a linguagem mais expressiva e clara. Como resultado, é proposto E-OntoUML, um perfil UML para a modelagem de processos usando diagramas de atividades, que é fundamentado semanticamente em UFO.
\end{abstract}

\begin{abstract}
Process models are used, among other things, to improve the operation and support of information systems development in organizations. In this context, the quality of the business process models is essential. Process models which are ambiguous and poorly formed can lead to problems of understanding and jeopardize their use. An important aspect for model quality regards the clarity and expressivity of the adopted modeling language. This paper explores the use of the Unified Foundational Ontology (UFO) to add real-world semantics to some model elements present in UML activity diagrams. As a result, we developed E-OntoUML, a UML profile for process modeling using activity diagrams that are semantically founded on UFO.
\end{abstract}

\section{Introdução}

A Gerência de Processos de Negócio (Business Process Management-BPM) baseia-se na observação de que produtos e serviços oferecidos pelas organizações são resultantes de processos de negócio. Ela tem recebido considerável atenção tanto da comunidade de administração quanto da comunidade de sistemas de informação: a primeira está interessada em aperfeiçoar a operação das empresas, levando em consideração aspectos como aumento da satisfação dos clientes, redução de custos e provimento de novos produtos e serviços a custos mais baixos; a segunda está interessada em prover sistemas de software robustos e escaláveis para apoiar processos de negócio [Weske 2007]. 
Sistemas de Informação (SIs) desempenham um importante papel na gerência de processos de negócio, já que cada vez mais atividades das organizações são apoiadas por sistemas desse tipo [Weske 2007]. Os SIs habilitam os processos de negócio e, se os processos de negócio e os sistemas não estiverem alinhados, então os SIs não atenderão às expectativas de seus usuários. Essa falta de alinhamento é apontada como sendo uma das principais causas de fracasso de projetos de desenvolvimento de SIs [Frye, Gulledge 2007].

Neste contexto, a qualidade dos modelos de processos de negócio é fundamental. Modelos de processo ambíguos e mal formados, por exemplo, podem levar a requisitos inconsistentes e, por conseguinte, a SIs que não atendam às necessidades do negócio.

O uso de notações gráficas para representar modelos de processo é reconhecido como essencial para sua apresentação de forma clara e concisa [Weske et al. 2004] e, por conseguinte, para o entendimento e comunicação do conhecimento nele expresso. Contudo, mesmo algumas das notações de modelagem de processos de negócio mais usadas na atualidade, como os diagramas de atividades da UML e BPMN [Ko et al. 2009], deixam margem para ambiguidades.

Este artigo explora o uso de uma ontologia de fundamentação para adicionar a chamada semântica de mundo real (ontological semantics ou real-world semantics) a alguns elementos de modelo de diagramas de atividade da UML, com vistas a deixar mais claros e menos ambíguos os modelos de processo de negócio elaborados utilizando essa notação. Uma ontologia de fundamentação é um sistema de categorias filosoficamente bem fundamentado e independente de domínio [Guizzardi et al. 2008]. Ontologias de fundamentação servem como base para o estabelecimento de consenso e negociação entre humanos, e têm sido utilizadas para melhorar a qualidade de linguagens de modelagem e modelos conceituais [Guizzardi 2005]. Neste trabalho, utiliza-se a ontologia de fundamentação UFO (Unified Foundational Ontology) [Guizzardi 2005] [Guizzardi et al. 2008], que possui conceitos relativos a eventos, objetos e agentes e, portanto, pode ser utilizada no contexto da modelagem de processos de negócio para prover semântica aos modelos que capturam o conhecimento dinâmico sobre processos. Consideramos a definição da semântica de mundo real complementar à definição de semântica formal de execução (como por exemplo aquela apresentada em [Eshuis 2002]). Enquanto a semântica de mundo real descreve uma linguagem em termos dos fenômenos que podem ser representados pelos elementos da linguagem, a semântica formal de execução define as possíveis execuções de um processo de negócio através de um mapeamento dos elementos da linguagem para um domínio semântico matemático (por exemplo, "tokens", "lugares", "transições" de uma rede de Petri).

Este artigo está organizado da seguinte forma. A seção 2 aborda a modelagem de processos de negócio e a qualidade de linguagens de modelagem para este propósito, bem como o papel de ontologias de fundamentação neste contexto. A seção 3 apresenta parcialmente E-OntoUML, um perfil UML para a modelagem de processos usando diagramas de atividades, que é fundamentado semanticamente em UFO. Conceitos de UFO e elementos de modelo da UML analisados são também apresentados nesta seção, de modo a mostrar a fundamentação usada no desenvolvimento do perfil proposto. Finalmente, as seções 4 e 5 apresentam, respectivamente, trabalhos correlatos e as conclusões deste trabalho. 


\section{Modelagem de Processos de Negócio}

Um modelo de processos de negócio descreve as atividades que uma organização executa para atingir os seus objetivos. Tipicamente, uma notação ou linguagem de modelagem de processos de negócio tem primitivas para descrever a ordenação e/ou causalidade entre eventos e atividades, assim como a participação de atores e recursos organizacionais nas atividades executadas.

De acordo com Ko et al. (2009), duas das notações mais amplamente utilizadas para representar modelos de processos de negócio são os diagramas de atividades da UML [OMG 2007] e diagramas de processos de negócio da BPMN (Business Process Modeling Notation) [OMG 2006]. White (2004) examinou esses dois tipos de diagramas considerando 21 padrões de workflow e concluiu que ambas as notações são adequadas para modelar a maioria dos padrões analisados. De acordo com White, as linguagens proveem soluções similares, compartilhando, inclusive, muitos símbolos comuns para o mesmo propósito. Russel et al. (2006) também examinaram a utilização de diagramas de atividades da UML para a modelagem de processos de negócio, concluindo que os diagramas de atividades podem ser utilizados para a modelagem de processos de negócio, ainda que eles não sejam adequados a todos os aspectos desse tipo de modelagem. Segundo esses autores, diagramas de atividades oferecem um bom suporte para as perspectivas de controle de fluxo e de dados, ainda que eles modelem aspectos organizacionais e relativos a recursos de forma limitada. Contudo, ainda de acordo com Russel et al. (2006), essas limitações são compartilhadas pela a maioria das linguagens de modelagem de processos de negócios e refletem a grande ênfase que tem sido dada ao controle de fluxo e à perspectiva de dados nas notações de modelagem de processos.

Neste trabalho optou-se por explorar os diagramas de atividades da UML como instrumento de modelagem de processos de negócio, tendo em vista que a UML é uma linguagem de modelagem amplamente utilizada no desenvolvimento de sistemas de informação. É importante realçar, contudo, que a modelagem de processos de negócios independe do desenvolvimento de sistemas e pode ser conduzida independentemente para a construção de uma arquitetura organizacional (enterprise architecture).

Um diagrama de atividades enfatiza a sequência e as condições que regem um comportamento, definindo seus fluxos de controle e de objetos [OMG 2007]. Para representar esses fluxos, a UML provê, dentre outros, elementos de modelos para representar ações, objetos, estados de objetos, fluxos de ações e de objetos, condições, eventos, início e fim de fluxo, exceções e agrupamento de ações.

Segundo Guizzardi (2007), um dos principais fatores de sucesso no uso de uma linguagem de modelagem reside na habilidade da linguagem prover aos usuários primitivas de modelagem que sejam capazes de expressar diretamente conceitos relevantes de um domínio, ou seja, de expressar a conceituação desse domínio. Assim, uma linguagem é o meio pelo qual se representa em símbolos (conjunto de elementos que compõem um vocabulário) as conceituações de uma realidade (ou de parte dela).

Para um modelo representar fidedignamente uma abstração, as primitivas de modelagem da linguagem usada para produzir esse modelo devem representar fidedignamente a conceituação usada para articular essa abstração. Quanto mais forte a 
correspondência entre uma abstração da realidade e seu modelo de representação, mais fácil é comunicar e raciocinar sobre esse modelo. A força dessa correspondência está diretamente ligada à qualidade da linguagem de modelagem usada [Guizzardi 2007].

Uma linguagem de modelagem delimita os possíveis modelos que podem ser construídos e, portanto, pode-se dizer que uma linguagem de modelagem ideal é aquela fiel ao seu domínio, ou seja, que tem como modelos válidos (gramaticalmente corretos) apenas os modelos que representam abstrações admissíveis pela conceituação daquele domínio. Isso é possível quando o metamodelo da linguagem de representação é isomórfico à ontologia ideal de domínio [Guizzardi 2007].

Tomando por base a argumentação de Guizzardi (2007), uma linguagem de modelagem de processos de negócio ideal deveria ter um metamodelo isomórfico a uma ontologia de fundamentação do domínio de processos. A UML, e por conseguinte seu diagrama de atividades, não foi construída com base em uma ontologia de fundamentação. Assim, seu metamodelo possui muitos conceitos estranhos a uma ontologia de fundamentação de processos.

Não é propósito deste trabalho propor uma linguagem de modelagem de processos ideal e, portanto, não se busca o isomorfismo em relação a uma ontologia de fundamentação. Busca-se apenas adicionar algumas distinções ontológicas consideradas importantes para a modelagem de processos de negócio, criando um perfil UML. Assim, utilizam-se os mecanismos de extensão da UML para adicionar parcialmente a semântica de uma ontologia de fundamentação a elementos de modelo de diagramas de atividades da UML. Dessa forma, busca-se estabelecer uma semântica de mundo real clara para esses elementos de modelo, por se comprometerem ontologicamente com uma ontologia de fundamentação. A ontologia de fundamentação usada é a Ontologia de Fundamentação Unificada (Unified Foundational Ontology - UFO) [Guizzardi 2005] [Guizzardi et al. 2008]. A UFO foi escolhida por unificar diversas ontologias de fundamentação e por estar sendo aplicada com sucesso para avaliar, (re)projetar e integrar modelos de linguagens de modelagem conceitual, assim como para prover semântica de mundo real para suas primitivas de modelagem [Guizzardi et al. 2008]. Alguns exemplos de uso de UFO para esses propósitos são apresentados na Seção 4, quando os mesmos são comparados com o presente trabalho.

A UFO é dividida em três partes complementares: a UFO-A é uma ontologia de indivíduos duradouros (endurants) e é o cerne da UFO; a UFO-B é uma ontologia de eventos (perdurants); por fim, a UFO-C é uma ontologia de entidades intencionais e sociais, construída sobre as partes A e B da UFO. As partes B e C da UFO tratam de conceitos relativos a eventos e entidades sociais, respectivamente, e, portanto, definem uma gama de conceitos importantes para a modelagem de processos. Uma vez que o enfoque deste trabalho é a modelagem de processos de negócio, em essência, são utilizadas essas partes de UFO. Os conceitos de UFO relevantes para este trabalho são apresentados na próxima seção, que discute o perfil UML proposto, de modo a mostrar a fundamentação usada no desenvolvimento do mesmo. Todas as definições da UFO usadas neste trabalho foram extraídas de [Guizzardi et al. 2008]. 


\section{E-OntoUML: Perfil UML para Diagramas de Atividades Fundamentado em UFO}

Um diagrama de atividades permite capturar o fluxo de controle entre atividades de um processo, isto é, a ordem de atividades, e suas condições de execução. Ele também permite modelar agentes que são responsáveis por executar essas atividades e as entradas e saídas das mesmas, especificando os estados anteriores e posteriores de cada objeto que participou de uma atividade. Entretanto, a UML é uma linguagem de especificação carente de uma semântica de mundo real bem definida. Os significados dos seus elementos de modelo são dados por descrições textuais e modelos que descrevem sua sintaxe abstrata. Assim, são frequentemente atribuídos diferentes significados a um mesmo elemento de modelo em diferentes situações. Em outras palavras, não há compromisso com distinções ontológicas importantes como as feitas em uma ontologia de fundamentação.

Visando tratar algumas distinções ontológicas importantes, foi desenvolvido EOntoUML, um perfil UML para a modelagem de processos usando diagramas de atividades, que é fundamentado semanticamente em UFO. E-OntoUML foi desenvolvido em partes, tratando dos seguintes aspectos da modelagem de processos:

- Ações: esta parte do perfil concentra-se em distinções relacionadas a diferentes tipos de atividades.

- Objetos: esta parte do perfil trata de tipos de objetos, estados dos objetos, entradas, saídas e tipos de participações de objetos em atividades.

- Agentes: esta parte do perfil aborda tipos de agentes e os tipos de participações de agentes em atividades.

- Fluxo de Controle: esta parte do perfil explora aspectos relacionados à ordenação e à sincronização de atividades.

Devido a limitações de espaço, não é possível discutir neste artigo todas as partes de E-OntoUML. Assim, a seguir discutem-se as partes relativas a ações e fluxo de controle.

Ações

Uma importante distinção ontológica de UFO é aquela entre indivíduos duradouros (Endurant) $)^{1}$ e eventos (Perdurant ou Event). Diz-se que indivíduos duradouros estão inteiramente presentes em qualquer instante do tempo em que estiverem presentes, enquanto eventos são indivíduos compostos de partes temporais. Em UFO, ações (Action) são eventos que tem o propósito específico de satisfazer alguma intenção de um agente. Ações podem ser atômicas (Atomic Action) ou complexas (Complex Action). Ações complexas são compostas de duas ou mais participações (Participation). Participações intencionais de agentes são denominadas participações de agentes $^{2}$ (Agent Participation) e uma ação complexa composta de participações de diferentes agentes é denominada uma interação (Interaction). Já uma

\footnotetext{
${ }^{1}$ Neste texto, conceitos da UFO aparecem destacados em negrito e itálico, enquanto elementos de modelo da UML são escritos em negrito e elementos do perfil E-OntoUML aparecem em negrito e sublinhados.

${ }^{2} \mathrm{O}$ termo originalmente empregado em [Guizzardi et al. 2008] para uma participação de agente é contribuição de ação (Action Contribution).
} 
participação de um objeto em uma ação é chamada de participação de objeto ${ }^{3}$ (Object Participation).

A UML, por sua vez, define ação como um elemento que é uma unidade fundamental para a execução de uma funcionalidade e atividade como uma sequência coordenada de ações [OMG 2007]. Para representar ações, a UML provê os símbolos mostrados na Figura 1. O primeiro é usado para representar uma ação (Action), que representa um único passo em uma atividade, isto é, algo que não é mais decomposto. Uma ação é simples do ponto de vista da atividade que a contém, mas pode ser complexa em seu efeito [OMG 2007]. Já o segundo símbolo corresponde a uma Ação de Chamada de Comportamento (CallBehaviorAction), o qual invoca um comportamento diretamente. Quando trata da chamada de uma atividade (Activity), está-se indicando que a mesma ocorre naquele ponto do fluxo e que suas ações são modeladas em outro diagrama, sendo a atividade, portanto, de natureza complexa e composta de ações ou outras atividades.

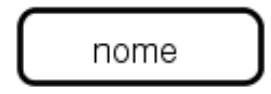

Ação

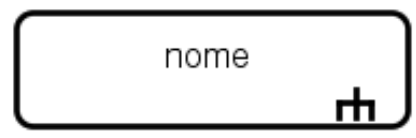

Ação de Chamada de Comportamento

Figura 1 - Elementos de modelo que representam ações e atividades na UML

Considerando a UFO e o metamodelo da UML, foram definidos em EOntoUML elementos de modelo capazes de capturar algumas das distinções ontológicas de UFO. A Figura 2 mostra uma visão parcial do metamodelo de E-OntoUML que trata de ações. Nesse modelo, conceitos da UFO são apresentados com o fundo cinza escuro, enquanto os elementos do metamodelo da UML estão em cinza claro. Os elementos de modelo do perfil E-OntoUML têm o fundo branco.

Conforme mostra a figura, o metamodelo da UML só permite capturar a distinção entre ações simples (não passíveis de decomposição) e atividades (as quais são decompostas em ações). Em outras palavras, o ponto de vista da UML é puramente mereológico. Outras distinções importantes feitas em UFO não são capturadas. Assim, em E-OntoUML foram criados outros elementos de modelo para capturar essas distinções.

Acão de agente (AgentAction) é uma especialização de ação (Action) da UML, assumindo a semântica definida em UFO, ou seja, uma ação de agente é representada pelo elemento de modelo ação (Action) da UML e é definida como um evento que muda o estado do mundo e é guiado pela intenção de um agente, conforme definido em UFO.

Uma ação (Action) em UFO pode ter um efeito complexo ou atômico, dependendo do número de participações (de agentes e de objetos) envolvidas nessa ação. Assim, foram criadas as especializações Ação de Agente Atômica (AtomicAgentAction) e Ação de Agente Complexa (ComplexAgentAction) para capturar essas distinções da UFO. Uma ação de agente atômica (AtomicAgentAction)

3 O termo originalmente empregado em [Guizzardi et al. 2008] para participações de objetos é participação de recurso (Resource Participation). 
especifica uma ação que envolve apenas uma participação de agente. Já uma ação de agente complexa (ComplexAgentAction) inclui ações que são complexas em efeito ou que podem ser divisíveis, podendo ser de dois tipos principais: interação (Interaction) e ação de agente com objeto (ObjectAgentAction). Uma interação (Interaction) envolve a participação de mais de um agente, sendo considerada de efeito complexo por agregar participações de vários agentes. Uma ação de agente com objeto (ObjectAgentAction), por sua vez, envolve a participação tanto de agentes quando de objetos. Logo, ela especifica uma ação complexa (ComplexAction) que é obrigatoriamente composta de pelo menos uma participação de objeto (ObjectParticipation) e de pelo menos uma participação de agente (Agent Participation). Vale observar que esses dois últimos elementos de E-OntoUML (interação e ação de agente com objeto), apesar de serem de natureza complexa, podem ou não envolver a decomposição de uma ação em subações (no sentido mereológico).

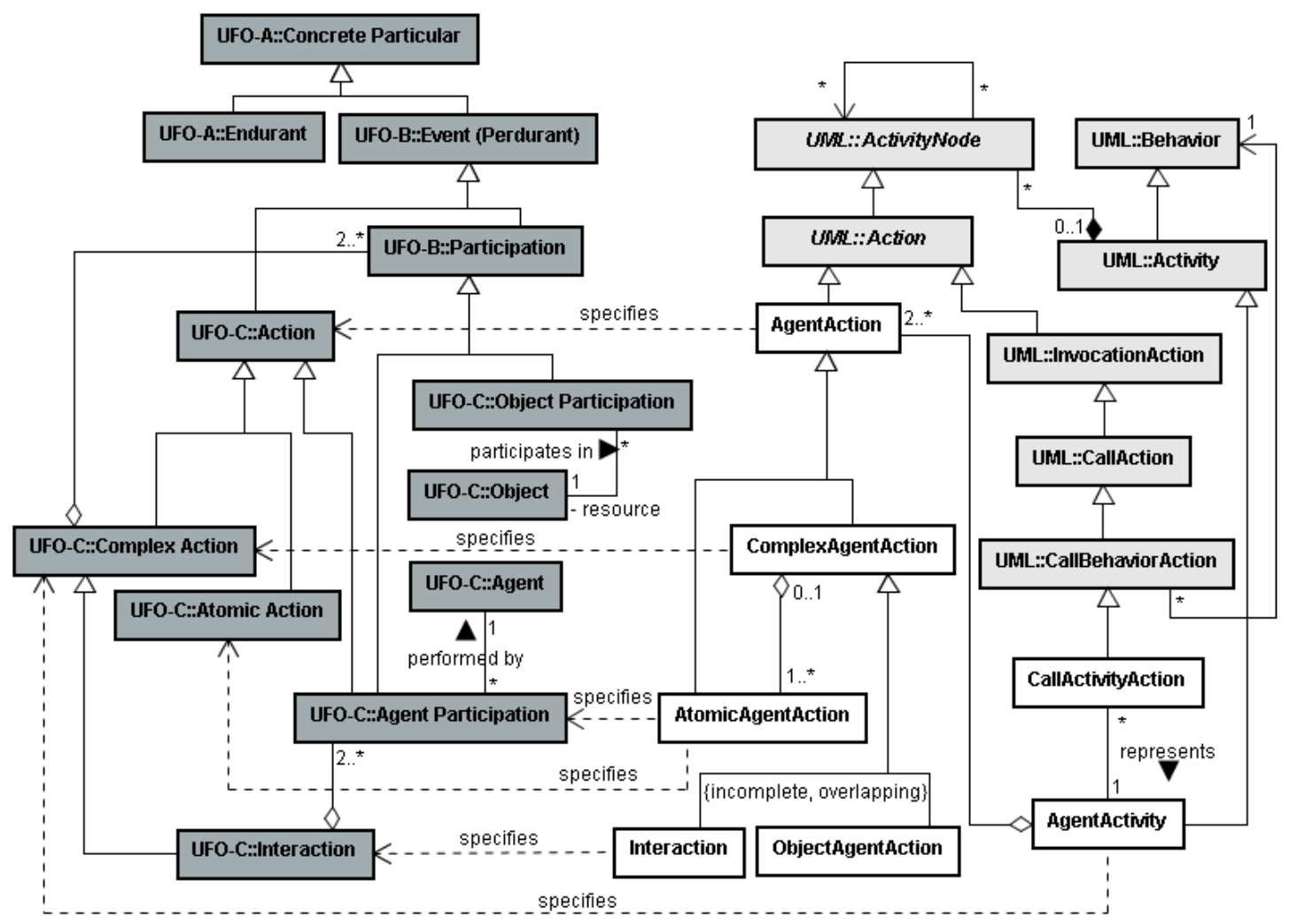

Figura 2 - Elementos de modelo de E-OntoUML para representar ações

Para representar ações que são decompostas em subações no sentido mereológico, o perfil E-OntoUML define o elemento atividade de agente (AgentActivity), o qual representa uma ação complexa não só no efeito, mas também pelo fato de ser decomposta em subações. Uma atividade de agente é composta de pelo menos duas accões de agente (AgentAction). Como citado anteriormente, uma atividade em um diagrama de atividades é graficamente representada pelo elemento de modelo ação de chamada de comportamento (CallBehaviorAction), logo foi necessário estender esse elemento para representar uma chamada a uma atividade de agente, sendo criado o elemento accão de chamada de atividade (CallActivityAction), que representa uma chamada a uma atividade de agente (AgentActivity). 
A Figura 3 mostra a notação gráfica de E-OntoUML para os elementos de modelo adicionados que possuem uma representação nos diagramas de atividades. A distinção entre ações decomponíveis e não passíveis de decomposição, no sentido mereológico, mantém-se inalterada em relação à UML e, portanto, os símbolos usados para ação de agente (AgentAction) e ação de chamada de atividade (CallActivityAction) são os mesmos de ação (Action) e ação de chamada de comportamento (CallBehaviorAction). Entretanto, para capturar a distinção entre uma ação complexa não passível de decomposição, no sentido mereológico, e uma ação atômica no sentido de UFO, i.e., que envolve uma e somente uma participação de agente, E-OntoUML propõe o estereótipo $<<$ atomic $>>$ para representar a segunda, ou seja, para representar uma ação de agente atômica (AtomicAgentAction).

Para capturar a distinção entre uma ação de agente realizada por um único agente e uma interacão (Interaction), envolvendo a participação de dois ou mais agentes, EOntoUML propõe o estereótipo <<interaction $>>$. Esse estereótipo pode ser usado tanto para ação não passível de decomposição, quanto para uma atividade. Ou seja, uma ação não passível de decomposição pode envolver a participação de vários agentes, assim como uma atividade decomposta em ações.

Por fim, uma accão de agente com objeto (ObjectAgentAction) é indicada pelo fato de envolver a participação de algum objeto, o que é representado por uma aresta ligando a ação a um nó de objeto (ObjectNode).
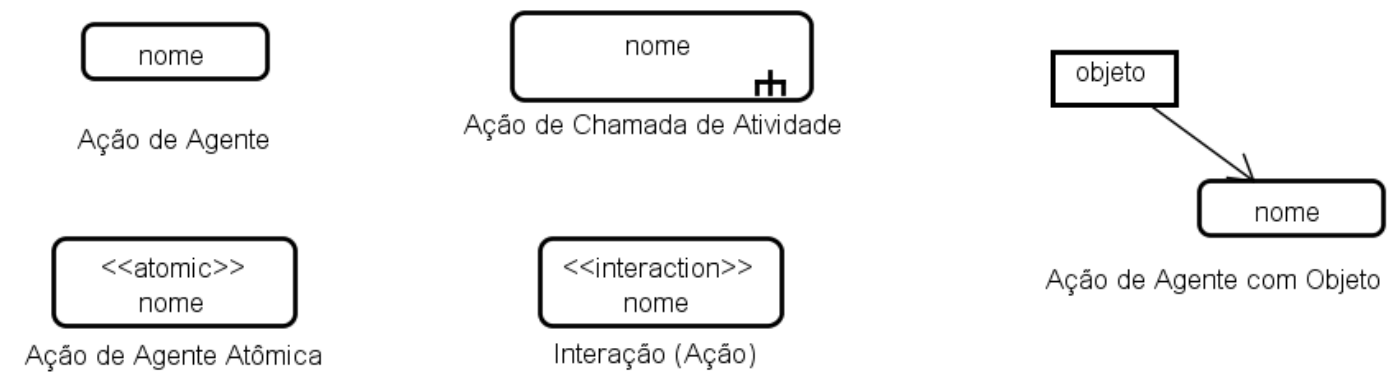

Ação de Agente com Objeto

Figura 3 - Elementos de modelo representando ações e atividades em E-OntoUML

A Figura 4 ilustra um exemplo de uso do perfil E-OntoUML para o caso de um processo de negócio de locação de carros. Este processo possui duas atividades principais (Locar Carro e Devolver Carro), as quais são ações complexas envolvendo subações. Assim, ambas as atividades são representadas usando a notação de chamada de atividade. Além disso, essas duas atividades envolvem a participação de dois agentes interagindo (o locatário e o locador) e de um objeto (o carro). Assim, ambas as ações são também interações e aç̃es de agente com objeto.

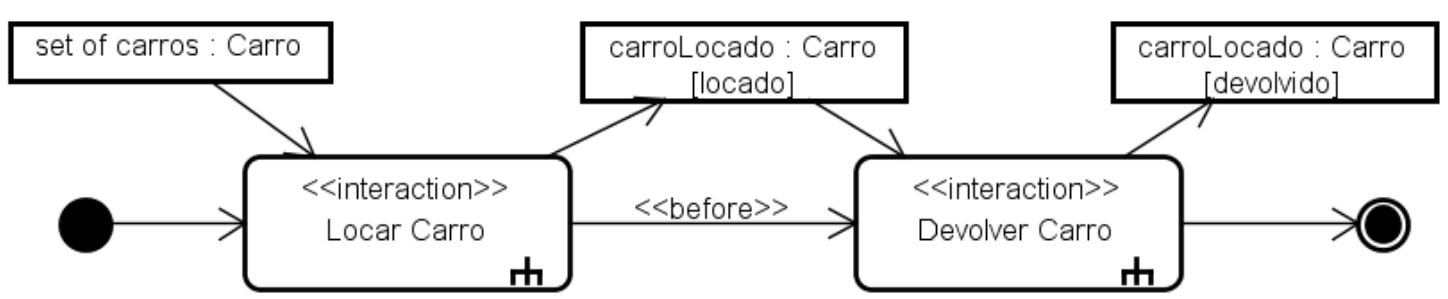

Figura 4 - Exemplo de uso do perfil E-OntoUML 


\section{Fluxo de Controle}

O fluxo de controle em diagramas de atividades da UML é modelado por arestas de atividades (ActivityEdge) denominadas fluxos de controle (ControlFlow). Um fluxo de controle (ControlFlow) indica o início de um nó de atividade destino (ActivityNode - target) após a conclusão de outro, dito origem (source), e é representado graficamente por uma seta ligando o nó origem ao nó destino. Assim, ele define a ordem de execução das ações que conecta. Contudo, ele não trata a relação temporal entre elas.

É possível aperfeiçoar este aspecto dos diagramas de atividades adicionando informações sobre as relações temporais existentes entre ações. A UFO, seguindo as definições de Allen (1983), estabelece sete relações temporais entre dois eventos, a saber: precede (before), encontra (meets), sobrepõe (overlaps), inicia (starts), durante (during), termina (finishes) e equivale (equals). A Figura 5 ilustra essas relações. As duas primeiras tratam de eventos sequenciais e as demais de eventos paralelos.

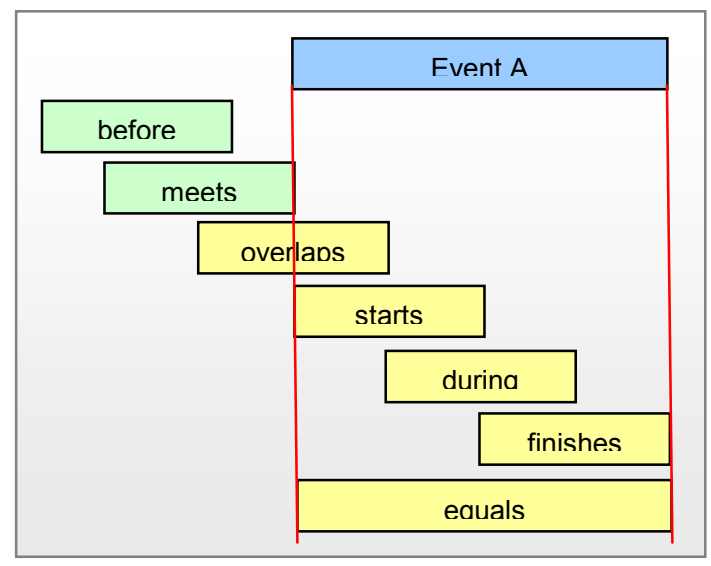

Figura 5 - Relações de Allen.

Para adicionar algumas dessas distinções ontológicas ao perfil E-OntoUML, a classe fluxo de controle (ControlFlow) do metamodelo da UML foi especializada para capturar a semântica das relações temporais, tendo sido criados os elementos de modelo mostrados na Figura 6. O elemento de modelo fluxo de controle de tempo (TimeControlFlow) trata de maneira geral uma relação temporal da UFO (Time Interval Relation). Esse elemento foi especializado em fluxo de controle sequencial (SequencialControlFlow), para tratar as relações sequenciais entre ações, as quais podem ser de dois tipos: (i) fluxo de controle de precedência (BeforeControlFlow) e (ii) fluxo de controle de encontro (MeetsControlFlow), que definem, respectivamente, as relações precede (before) e encontra (meets) de Allen. A representação desses elementos é feita pela adição de um estereótipo ao fluxo de controle (ControlFlow), indicando qual a relação temporal que está sendo considerada $(<<$ before $>>$ ou $<<$ meets $>>$ ). No exemplo da Figura 4, a relação temporal entre as atividades Locar Carro e Devolver Carro é de precedência e, portanto, foi utilizado o estereótipo $<<$ before $>>$.

As cinco demais relações temporais citadas envolvem paralelismo entre ações e a UML não possui uma primitiva de modelagem capaz de representá-las diretamente. Para representar ações que ocorrem em paralelo na UML, é necessário utilizar nós de 
controle (ControlNode), um tipo de nó de atividade (ActivityNode) usado para coordenar o fluxo de controle entre outros nós [OMG 2007]. Contudo, mesmo usando nós de atividade, não é possível capturar as distinções entre as relações sobrepõe (overlaps), inicia (starts), durante (during), termina (finishes) e equivale (equals). Um perfil (lightweight extension), como é o caso de E-OntoUML, não tem como adicionar as restrições necessárias, sendo necessária uma revisão do metamodelo da UML.

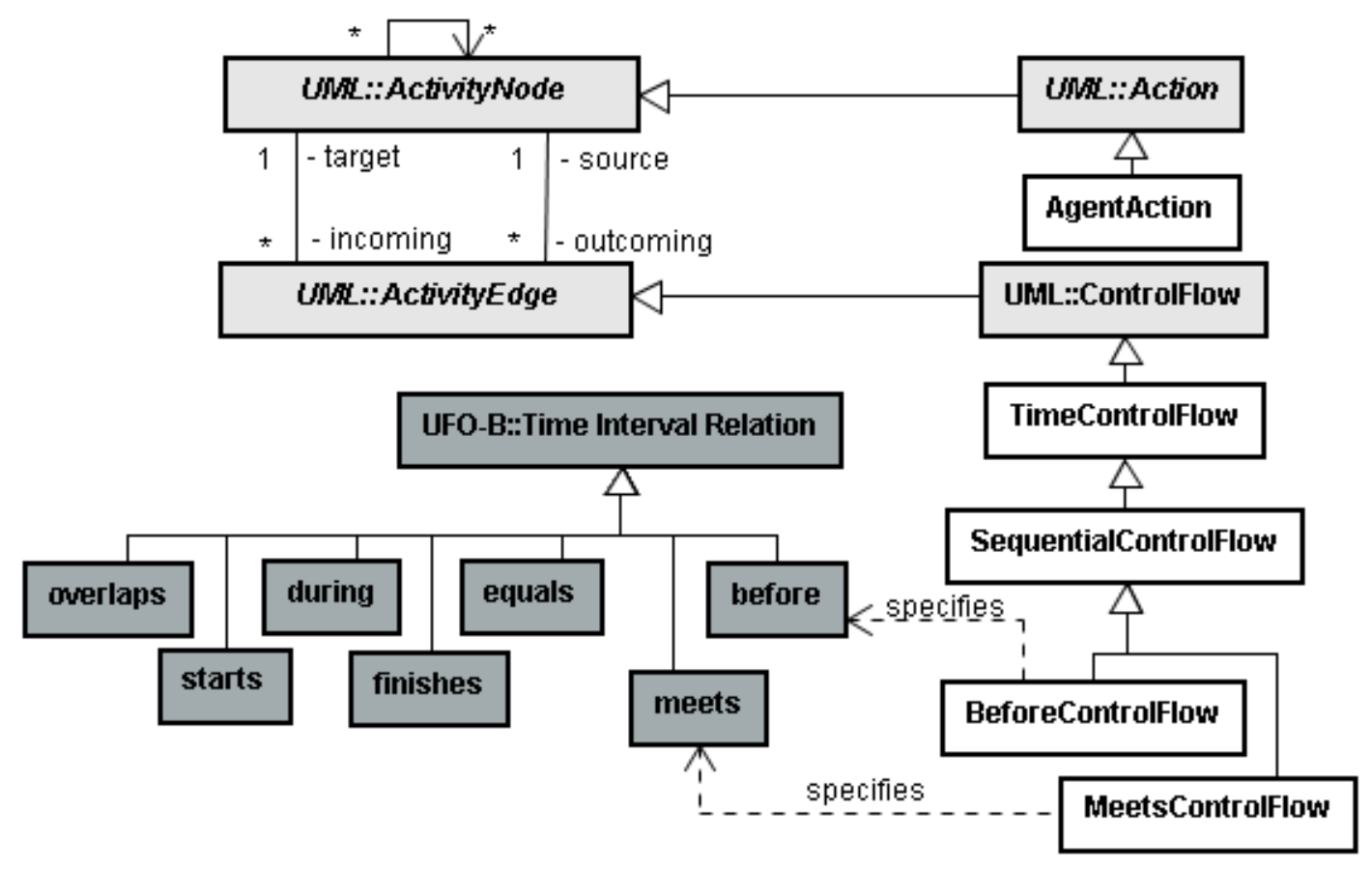

Figura 6 - Elementos de modelo de E-OntoUML relativos a fluxos de controle

\section{Trabalhos Correlatos}

Há diversos trabalhos relacionados ao uso de ontologias de fundamentação para avaliar e reprojetar modelos de linguagens de modelagem conceitual, bem como para prover semântica de mundo real para seus elementos de modelo. Guizzardi (2005), por exemplo, utilizou a ontologia de fundamentação UFO-A para avaliar o metamodelo da UML relativo à parte que trata das primitivas de modelagem usadas na construção de diagramas de classes e definir o perfil OntoUML. Este trabalho seguiu estratégia análoga, no sentido de analisar o metamodelo da UML (a parte comportamental relativa a diagramas de atividades) e propor um perfil UML.

$\mathrm{Na}$ área de modelagem de processos de negócio, Almeida, Guizzardi e colegas têm diversos trabalhos enfocando, entre outros, a fundamentação semântica de conceitos relacionados a papéis [Almeida et al. 2009] e a análise ontológica de linguagens de modelagem propostas pelo método ARIS, dentre elas a linguagem de modelagem de processos EPC (Event-driven Process Chain) [Santos Jr. et al. 2010]. Em todos esses trabalhos é utilizada como base para a análise ontológica a Ontologia de Fundamentação Unificada (UFO) e, neste sentido, o presente trabalho está bastante em linha com os trabalhos citados. Em especial, o trabalho de Santos Jr. et al. (2010) é bastante relacionado ao presente trabalho, uma vez que ambos tratam de linguagens de modelagem de processo. Contudo, em [Santos Jr. et al. 2010] é feita apenas uma análise 
ontológica para prover uma interpretação ontológica dos elementos de modelo em diagramas EPC em termos da UFO. Este trabalho segue uma linha um pouco diferente e utiliza os mecanismos de extensão da UML para desenvolver um perfil UML para diagramas de atividades, o qual é fundamentado em conceitos da UFO.

Outros trabalhos têm usado outras ontologias de fundamentação para efetuar análise ontológica de linguagens de modelagem de processos, como é o caso de [Green, Rosemann 2005], em que os autores fazem uma análise ontológica de diagramas EPC em termos de uma ontologia de fundamentação denominada BWW (Bunge-WandWeb). Porém, no melhor de nosso conhecimento, para a parte comportamental da UML, mais especificamente no que se refere à parte que trata de diagramas de atividades, não foram encontrados análises ontológicas ou perfis estabelecidos com base em uma ontologia de fundamentação.

\section{Conclusões}

Atualmente, reconhece-se a Gerência de Processos de Negócio como uma área de grande importância tanto para a melhoria das organizações quanto como uma ferramenta útil para o desenvolvimento de sistemas de informação. Neste contexto, cresce o interesse na questão da qualidade dos modelos de processos e, por conseguinte, das linguagens de modelagem usadas para representá-los. Este trabalho apresentou parcialmente um perfil UML para a modelagem de processos usando diagramas de atividades fundamentado na Ontologia de Fundamentação Unificada (Unified Foundational Ontology - UFO). Esse perfil adiciona semântica de mundo real a alguns elementos de modelo de diagramas de atividade da UML, com vistas a deixar mais claros e menos ambíguos os modelos de processo de negócio elaborados utilizando essa notação.

\section{Agradecimentos}

Este trabalho foi financiado pelas agências de fomento à pesquisa FAPES (Processo Número 45444080/09) e CNPq (Processos Número 481906/2009-6, 309059/2008-9 e 483383/2010-4).

\section{Referências}

Allen, J.F. (1983) "Maintaining Knowledge about Temporal Intervals", Communications of the ACM, v. 26, issue 11, pp. 832-843.

Almeida, J.P.A., Guizzardi, G., Santos Jr., P.S. (2009). “Applying and Extending a Semantic Foundation for Role-Related Concepts in Enterprise Modelling”. Enterprise Information Systems, v. 3, p. 253-277, 2009.

Eshuis, H. (2002) Semantics and Verification of UML Activity Diagrams for Workflow Modelling. Ph.D. Thesis, University of Twente, The Netherlands.

Frye, D. W., Gulledge, T. R. (2007) "End-to-end Business Process Scenarios". Industrial, Management \& Data Systems, v. 107, n. 6, pp.749-761.

Green, P.F., Rosemann, M. (2005) Ontological Analysis of Business Systems Analysis Techniques: Experiences and Proposals for and Enhanced Methodology. In Business Systems Analysis with Ontologies, IGI Global, pp 1- 27. 
Guizzardi, G. (2005) Ontological Foundations for Structural Conceptual Models, Universal Press, The Netherlands.

Guizzardi, G. (2007) "On Ontology, ontologies, Conceptualizations, Modeling Languages, and (Meta)Models", Frontiers in Artificial Intelligence and Applications, Databases and Information Systems IV, Olegas Vasilecas, Johan Edler, Albertas Caplinskas (Editors), IOS Press, Amsterdam.

Guizzardi, G., Falbo, R.A. Guizzardi, R.S.S. (2008) "A importância de Ontologias de Fundamentação para a Engenharia de Ontologias de Domínio: o caso do domínio de Processos de Software", IEEE Latin America Transactions, v. 6, no. 3, p. 244-251.

Ko, R.K., Lee, S.S., Lee, E.W. (2009) "Business process management (BPM) standards: A survey”, Business Process Management Journal, vol. 15, Issue 5, pp.744 - 791.

Lu, R., Sadiq, S. (2007) "A survey of comparative business process modeling approaches", In: Abramowicz, W., editor, Business Information Systems, Lecture Notes in Computer Science, vol. 4439, p. 82-94, Springer Berlin / Heidelberg.

OMG (2006) Business Process Modeling Notation Specification, Final Adopted Specification, February.

OMG (2007) OMG Unified Modeling Language (OMG UML), Superstructure V2.1.2, formal/2007-11-02, November.

Russell, N., Van der Aalst, W.M.P., Ter Hofstede, A.H.M., Wohed, P. (2006) "On the suitability of UML 2.0 activity diagrams for business process modeling", Proceedings of the 3rd Asia-Pacific Conference on Conceptual Modeling, Hobart, Australia, pp. 95-104.

Santos Jr., P.S., Almeida, J.P.A., Guizzardi, G. (2010) "An Ontology-based Semantic Foundation for ARIS EPCs", In: Proceedings of the 25th ACM Symposium on Applied Computing (Enterprise Engineering Track), 2010, Sierre, Switzerland.

Wand, Y., Weber, R. (1989) "An ontological evaluation of systems analysis and design methods". In: Information System Concepts: An In-Depth Analysis. Elsevier Science Publishers B.V., North-Holland.

Weske, M. (2007) Business Process Management: Concepts, Languages, Architectures. Springer, $1^{\text {st }}$ edition.

Weske, M., Van der Aalst, W.M.P., Verbeek, H.M.W. (2004) "Advances in business process management". Data \& Knowledge Engineering, 50(1):1-8.

White, S.A. (2004) Process Modeling Notations and Workflow Patterns, BPTrends. 